\title{
CLASSIFICAÇÃO DA COBERTURA DO SOLO URBANO USANDO ÁRVORES DE DECISÃO A PARTIR DE UMA CENA WORLDVIEW-2 PARA DIFERENTES NÍVEIS DE LEGENDA
}

\author{
DECISION TREES-BASED URBAN LAND COVER CLASSIFICATION OF WORLDVIEW-2 \\ SCENES AT DIFFERENT LEGEND LEVELS
}

\author{
Paulo Roberto da Silva RUIZ ${ }^{1}$, Cláudia Maria de ALMEIDA ${ }^{1}$, Camila Souza dos ANJOS ${ }^{2}$ \\ ${ }^{1}$ Instituto Nacional de Pesquisas Espaciais - INPE. Divisão de Sensoriamento Remoto. São José dos Campos, Brasil \\ Email: paulo.ruiz@inpe.br; almeida@dsr.inpe.br \\ ${ }^{2}$ Instituto de Estudos Avançados - IEAv. Subdivisão de Sensoriamento Remoto. Força Aérea Brasileira. São José dos Campos, Brasil
} Email: camilasanjos@gmail.com

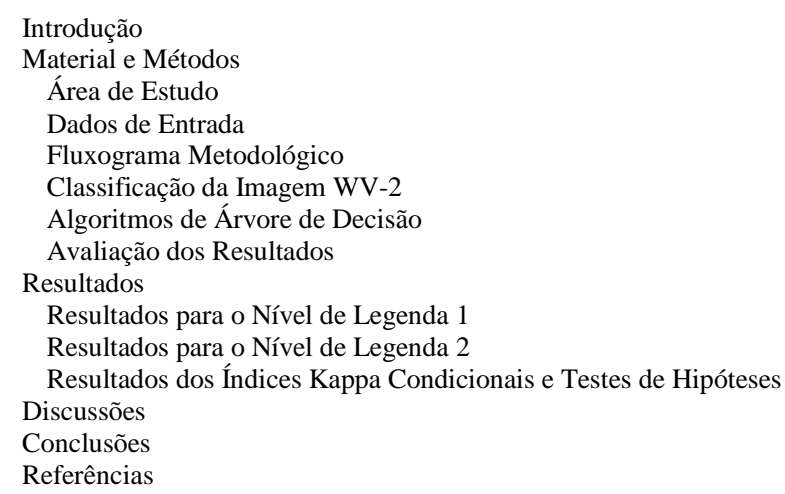

RESUMO - No presente trabalho, foram realizadas classificações da cobertura do solo urbano a partir de uma imagem WorldView2, sendo realizadas para dois níveis de legenda de classificação, contendo respectivamente 11 e 38 classes de cobertura do solo. Para isto, foram utilizados os algoritmos de árvore de decisão Best First Tree (BFTree) e Logistic Model Tree (LMT). Os resultados foram validados por meio de mapas de verdade de campo da área de estudo, revelando que o algoritmo LMT obteve os melhores resultados em ambos os níveis de detalhamento.

Palavras-chave: classificação de cobertura do solo, sensoriamento remoto urbano, WorldView-2, mineração de dados.

ABSTRACT - In this work, urban land cover classification was performed using a WorldView-2 image, carried out at two legend levels, respectively containing 11 and 38 land cover classes. As classification methods, we used the Best First Tree (BFTree) and Logistic Model Tree (LMT), which correspond to decision tree induction algorithms. The results were validated using data collected on field trips, revealing that the LMT algorithm obtained the best results at both levels of legend.

Keywords: land cover classification, urban remote sensing, WorldView-2, data mining.

\section{INTRODUÇÃO}

A partir de 2007, a humanidade testemunhou a superação do contingente de população rural pelo contingente de população urbana (UNPD, 2014). No caso do Brasil, a população urbana supera o índice de 84\% (IBGE, 2013). Portanto, o crescimento populacional urbano é um fenômeno que ocorre com grande rapidez, sendo considerado um processo irreversível. Com isso, as cidades necessitam de uma melhora no planejamento urbano para atender às necessidades dos cidadãos quanto à qualidade de vida, à distribuição equitativa de bens e serviços e à sua inclusão socioeconômica (BRASIL, 2002).

Para contribuir com o desenvolvimento de estudos urbanos voltados ao planejamento das cidades, são necessárias novas fontes de dados. Nesse contexto, o sensoriamento remoto ganha destaque por possibilitar o mapeamento e a atualização cartográfica dessas áreas. A melhora sistemática da qualidade geométrica dos sensores orbitais de alta resolução espacial e o imageamento em vários canais multiespectrais permitem detalhar os alvos urbanos. Esses recursos são requeridos para o mapeamento urbano, pelo fato de as cidades possuírem uma grande diversidade de alvos, geralmente de pequeno e médio porte (Jensen, 2011).

Mas a disponibilização de altas resoluções espaciais e espectrais significaram novos desafios para o sensoriamento remoto, uma vez que o refinamento de ambas as resoluções exige melhores recursos computacionais para o processamento, manipulação e armazenamento dessas imagens. Nesse sentido, o presente trabalho propõe-se a explorar as potencialidades e limitações do sensor WorldView-2 (WV-2) para a classificação de alvos urbanos 
em diferentes níveis de legenda.

O termo nível de legenda de classificação (em inglês, legend level) é amplamente utilizado na literatura para se referir ao nível de detalhamento de uma classificação, expresso invariavelmente pelo número de classes constantes da legenda (Herold et al., 2004; Jensen, 2011). Para isso, serão utilizados métodos não paramétricos de classificação de imagens baseados em árvores de decisão.

\section{MATERIAL E MÉTODOS}

Esta seção apresenta a área de estudo, o material e a metodologia utilizada em seu desenvolvimento.

\section{Área de Estudo}

A área de estudo situa-se na cidade de Campinas, interior paulista, a qual possui as seguintes características: coordenada central de 2254'3'”S e 47³'26' 'W, altitude média de $685 \mathrm{~m}$, superfície de $794,571 \mathrm{~km}^{2}$, população estimada em 2015, ano de realização desta pesquisa, de 1.164.098 habitantes e constitui-se no maior polo tecnológico da América Latina (IBGE, 2014).

Um transecto do campus da UNICAMP foi escolhido para a realização do presente trabalho. A área desse campus atinge quase 3,4 mil quilômetros quadrados e está repleta de prédios rodeados por parques e gramados (UNICAMP, 2016).

A diversidade de alvos urbanos, a fácil acessibilidade aos edifícios e a disponibilidade de dados para este local foram as razões para a sua escolha nesta pesquisa (Figura 1).

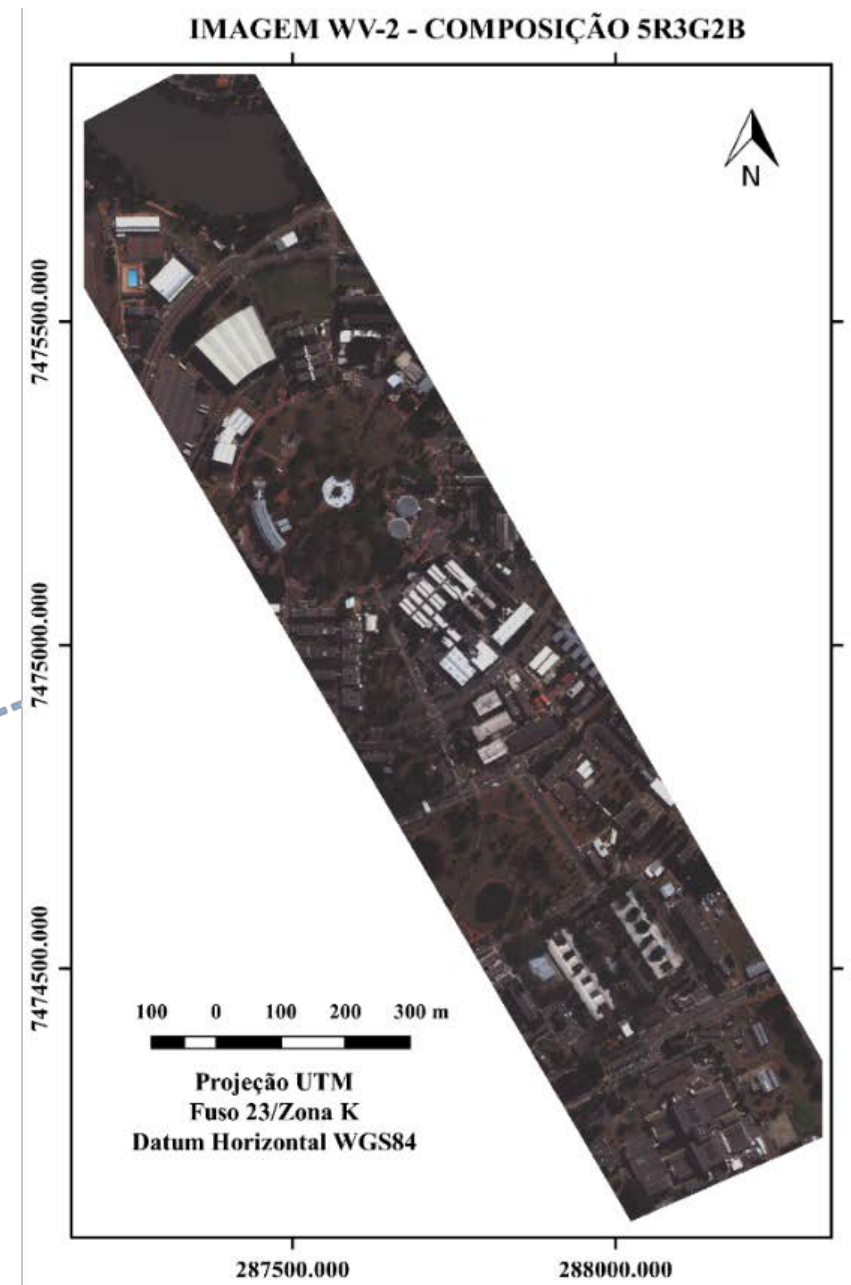

Figura 1 - Localização da área de estudo. Fonte: Adaptado de Google (2015).

\section{Dados de Entrada}

O trabalho foi realizado a partir de uma imagem orbital do sensor WorldView-2 adquirida em 02/07/2010, com angulação de $6,75^{\circ}$ off nadir, $38,37^{\circ}$ de elevação solar e $0 \%$ de cobertura de nuvens. Este sensor possui resolução espacial no nadir de $0,46 \mathrm{~m}$ no modo pancromático e 1,84 m no multiespectral. As imagens são quantizadas em 11 bits de resolução radiométrica e possui, além dos canais espectrais convencionais do azul (450$510 \mathrm{~nm})$, verde (510-580 nm), vermelho 
(630-690 nm) e infravermelho próximo (750$745 \mathrm{~nm})$, bandas adicionais do azul costeiro (400-450 nm), amarelo (585-625 nm), vermelho limítrofe (705-745 $\mathrm{nm})$ e infravermelho próximo 2 (860-1040 nm) (DIGITALGLOBE, 2009).

\section{Fluxograma Metodológico}

A Figura 2 apresenta o fluxograma metodológico deste trabalho. As etapas de pré-processamento, extração de atributos e segmentação da imagem (WV-2) foram realizadas no trabalho de Anjos (2016).

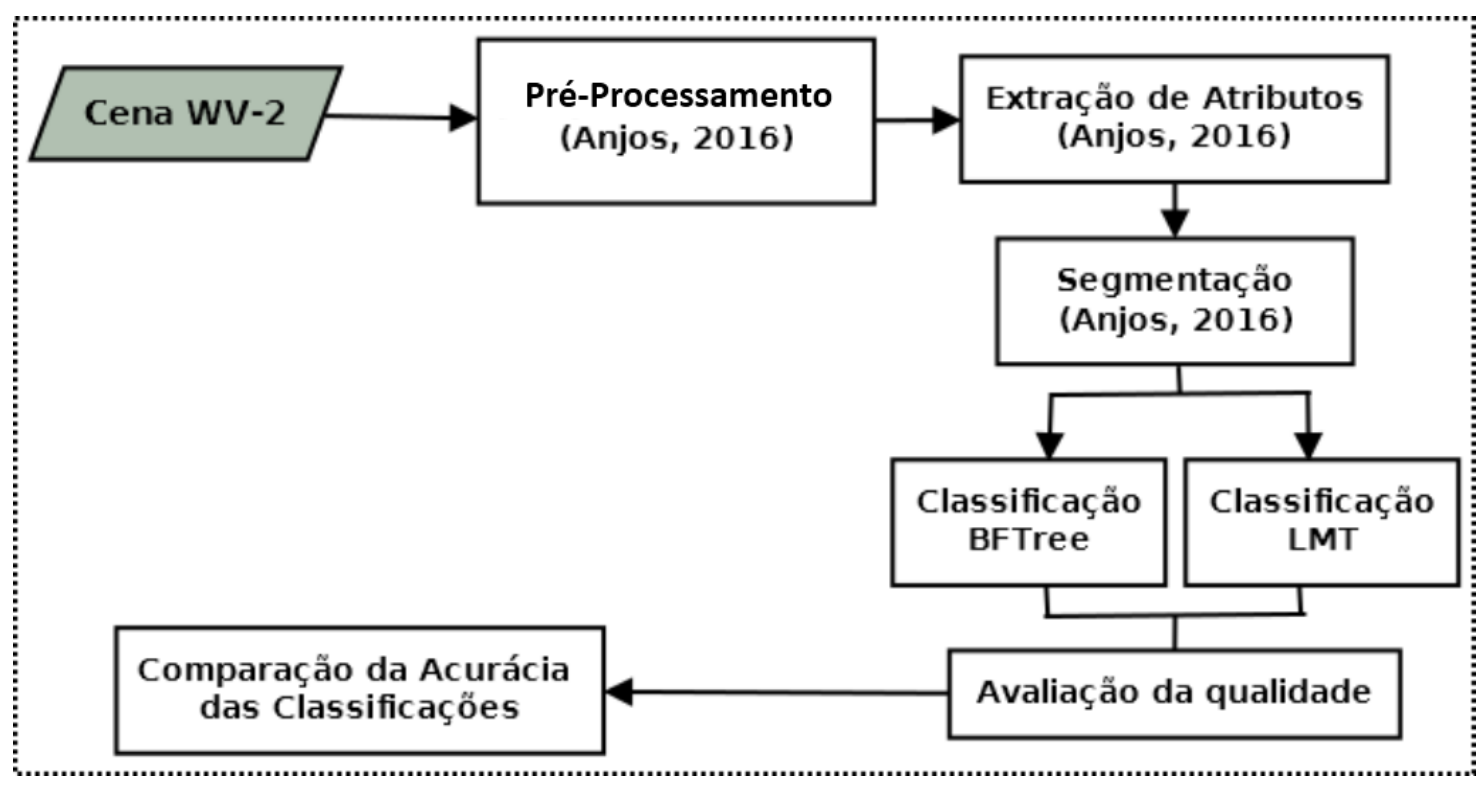

Figura 2 - Fluxograma das etapas metodológicas do trabalho.

O pré-processamento envolve a correção atmosférica, realizada por meio do algoritmo FLAASH (Fast Line-of-sight Atmospheric Analysis of Spectral Hypercubes ou "Análise Rápida de Linha de Visão Atmosférica de Hipercubos Espectrais") do ENVI 5.1 (SULSOFT, 2000) e a ortorretificação, realizada por meio da ferramenta "Dispositivo de Ortorretificação” (OrthoEngine) do PCI Geomatics (GEOMATICA, 2014).

Os atributos utilizados foram: Análise por Componentes Principais ou Principal Component Analysis (PCA); Fração de Ruído Mínima ou Minimum Noise Fraction (MNF); Brilho ou Brightness; Modelo Digital de Altura (MDA), que é resultante da subtração entre o Modelo Digital de Superfície (MDS) e o Modelo Digital de Terreno (MDT); quatro Índices de Vegetação por Diferença Normalizada (NDVI), alternando-se as bandas do vermelho e vermelho limítrofe, bem como as bandas do infravermelho próximo 1 e 2; Índice de Vegetação Ajustado ao Solo (SAVI); além de 19 atributos customizados (ACs), obtidos por meio de aritmética de bandas do WV-2, apresentados na Tabela 1 (Anjos, 2016).

As equações apresentadas na Tabela 1 utilizam as seguintes bandas WV-2: B2 - azul (centrada em 480 nm), B3 - verde (centrada em 545 nm), B4 - amarelo (centrada em $605 \mathrm{~nm}$ ), B5 vermelho (centrada em $660 \mathrm{~nm}$ ) e B7 infravermelho próximo (centrada em $832 \mathrm{~nm}$ ). Estes dados apresentam outras bandas multiespectrais: B1 - azul costeiro (centrada em 425 nm), B6 - vermelho limítrofe (centrada em $725 \mathrm{~nm}$ ), além de outra banda na região do infravermelho próximo: B8 - infravermelho próximo 2 (centrada em $950 \mathrm{~nm}$ ).

Com isso, por meio de combinações lineares, outras equações foram adaptadas, perfazendo o total de 19 atributos customizados (Anjos, 2016).

Como foram desenvolvidas classificações da cobertura do solo urbano baseadas em regiões, é necessária a realização da segmentação da imagem. Esse procedimento foi realizado por meio dos recursos avançados do algoritmo de segmentação multirresolução, disponível no software eCognition Developer 8.7 (TRIMBLE, 2011).

Após a segmentação, foi executado o algoritmo de ajuste por diferença espectral (Spectral Difference), que é utilizado para refinar os resultados da segmentação através da similaridade espectral entre os segmentos vizinhos (DEFINIENS, 2007). 
Tabela 1 - Atributos utilizados nas classificações.

\begin{tabular}{|c|c|c|}
\hline ATRIBUTO & EQUAÇÕES & REFERÊNCIA \\
\hline MDA & $M D A=\mathrm{MDS}-\mathrm{MDT}$ & Leonardi (2010) \\
\hline BRIGHTNESS & Brightness $=\frac{\sum_{i=1}^{n} \text { banda } i}{n}$ & DEFINIENS (2007) \\
\hline NDVI 1 & NDVI $1=\frac{\text { Banda }_{832 n m}-B a n d a_{660 n m}}{B a n d a_{832 n m}+B a n d a_{660 n m}}$ & Rouse et al. (1974) \\
\hline NDVI 2 & NDVI $2=\frac{\text { Banda }_{832 \mathrm{~nm}}-\text { Banda }_{725 \mathrm{~nm}}}{\text { Banda }_{832 \mathrm{~nm}}+\text { Banda }_{725 \mathrm{~nm}}}$ & Adaptado de Rouse et al. (1974) \\
\hline NDVI 3 & NDVI $3=\frac{\text { Band }_{950 \mathrm{~nm}}-\text { Banda }_{660 \mathrm{~nm}}}{\text { Band }_{950 \mathrm{~nm}}+B a n d a_{660 \mathrm{~nm}}}$ & Adaptado de Rouse et al. (1974) \\
\hline NDVI 4 & NDVI $4=\frac{\text { Band }_{950 \mathrm{~nm}}-\text { Banda }_{725 \mathrm{~nm}}}{\text { Band }_{950 \mathrm{~nm}}+\text { Banda }_{725 \mathrm{~nm}}}$ & Adaptado de Rouse et al. (1974) \\
\hline SAVI & $\mathrm{SAVI}=\frac{(1+L)\left(\text { Banda }_{832 \mathrm{~nm}}-B a n d a_{660 \mathrm{~nm}}\right)}{B a n d a_{832 \mathrm{~nm}}+B a n d a_{660 \mathrm{~nm}}+L}$ & Huete (1988) \\
\hline AC 1 & $\mathrm{AC} 1=\frac{\text { Brightness }+ \text { Banda }_{480 \mathrm{~nm}}}{\text { Banda }_{660 \mathrm{~nm}}}$ & Leonardi (2010) \\
\hline AC 2 & AC $2=\frac{\text { Brightness }+ \text { Banda }_{480 n m}}{\text { Banda }_{725 n m}}$ & Adaptado de Leonardi (2010) \\
\hline AC 3 & $A C 3=$ Brightness $+B a n d a_{480 \mathrm{~nm}}-B a n d a_{660 \mathrm{~nm}}$ & Adaptado de Leonardi (2010) \\
\hline $\mathrm{AC} 4$ & $A C 4=$ Brightness $+B a n d a_{480 \mathrm{~nm}}-B a n d a_{725 \mathrm{~nm}}$ & Adaptado de Leonardi (2010) \\
\hline AC 5 & $A C 5=$ Brightness - Banda $a_{832 n m}$ & Adaptado de Leonardi (2010) \\
\hline AC 6 & AC $6=$ Brightness - Banda $_{950 n m}$ & Adaptado de Leonardi (2010) \\
\hline AC 7 & $\mathrm{AC} 7=\frac{\text { Banda }_{660 \mathrm{~nm}}}{\text { Banda }_{832 \mathrm{~nm}}}$ & Richardson \& Wiegand (1977) \\
\hline AC 8 & $\mathrm{AC} 8=\frac{\text { Banda }_{725 \mathrm{~nm}}}{\text { Banda }_{832 \mathrm{~nm}}}$ & $\begin{array}{c}\text { Adaptado de Richardson \& Wiegand } \\
\text { (1977) }\end{array}$ \\
\hline AC 9 & AC $9=\frac{B a n d a_{660 \mathrm{~nm}}}{B a n d a_{950 \mathrm{~nm}}}$ & $\begin{array}{l}\text { Adaptado de Richardson \& Wiegand } \\
\text { (1977) }\end{array}$ \\
\hline AC 10 & $\mathrm{AC} 10=\frac{\text { Banda }_{725 \mathrm{~nm}}}{\text { Band }_{950 \mathrm{~nm}}}$ & $\begin{array}{c}\text { Adaptado de Richardson \& Wiegand } \\
\text { (1977) }\end{array}$ \\
\hline AC 11 & AC $11=\frac{\text { Banda }_{545 \mathrm{~nm}}}{\text { Band }_{832 \mathrm{~nm}}}$ & Bausch \& Duke (1996) \\
\hline AC 12 & $\mathrm{AC} 12=\frac{\text { Banda }_{725 \mathrm{~nm}}}{\text { Band }_{832 \mathrm{~nm}}}$ & Adaptado de Bausch \& Duke (1996) \\
\hline AC 13 & AC $13=\frac{\text { Banda }_{832 n m}-\text { Banda }_{545 \mathrm{~nm}}}{\text { Banda }_{832 \mathrm{~nm}}+B a n d a_{545 \mathrm{~nm}}}$ & Gitelson et al. (1996) \\
\hline AC 14 & AC $14=\frac{\text { Banda }_{950 \mathrm{~nm}}-\text { Banda }_{545 \mathrm{~nm}}}{\text { Banda }_{950 \mathrm{~nm}}+B a n d a_{545 \mathrm{~nm}}}$ & Adaptado de Gitelson et al. (1996) \\
\hline AC 15 & $\mathrm{AC} 15=\frac{\text { Brightness }+ \text { Banda }_{425 \mathrm{~nm}}}{\text { Banda }_{660 \mathrm{~nm}}}$ & Adaptado de Rouse et al. (1974) \\
\hline AC 16 & $\mathrm{AC} 16=\frac{\text { Brightness }+ \text { Band } a_{425 \mathrm{~nm}}}{\text { Banda }_{725 \mathrm{~nm}}}$ & Adaptado de Leonardi (2010) \\
\hline AC 17 & $A C 17=$ Brightness + Banda $_{480 \mathrm{~nm}}-$ Banda $_{725 \mathrm{~nm}}$ & Adaptado de Leonardi (2010) \\
\hline AC 18 & $A C 18=$ Brightness + Banda $_{480 \mathrm{~nm}}-$ Banda $_{832 \mathrm{~nm}}$ & Adaptado de Leonardi (2010) \\
\hline AC 19 & AC $19=\frac{\text { Band }_{545 n m}-B a n d a_{832 n m}}{B a n d a_{545 n m}+B a n d a_{832 n m}}$ & $\begin{array}{l}\text { Jain et al. (2006) } \\
\text { Sarkar et al. (2012) }\end{array}$ \\
\hline
\end{tabular}

\section{Classificação da Imagem WV-2}

Primeiramente, é necessária a definição das classes de interesse, sua estruturação em diferentes níveis de legenda e as respectivas chaves de interpretação. Neste sentido, a hierarquia de classes de cobertura do solo urbano foi apresentada no trabalho de Anjos (2016). A Tabela 2 apresenta os níveis de legenda de classificação utilizados neste trabalho. 
Tabela 2. Níveis de legenda de classificação da cobertura do solo urbano.

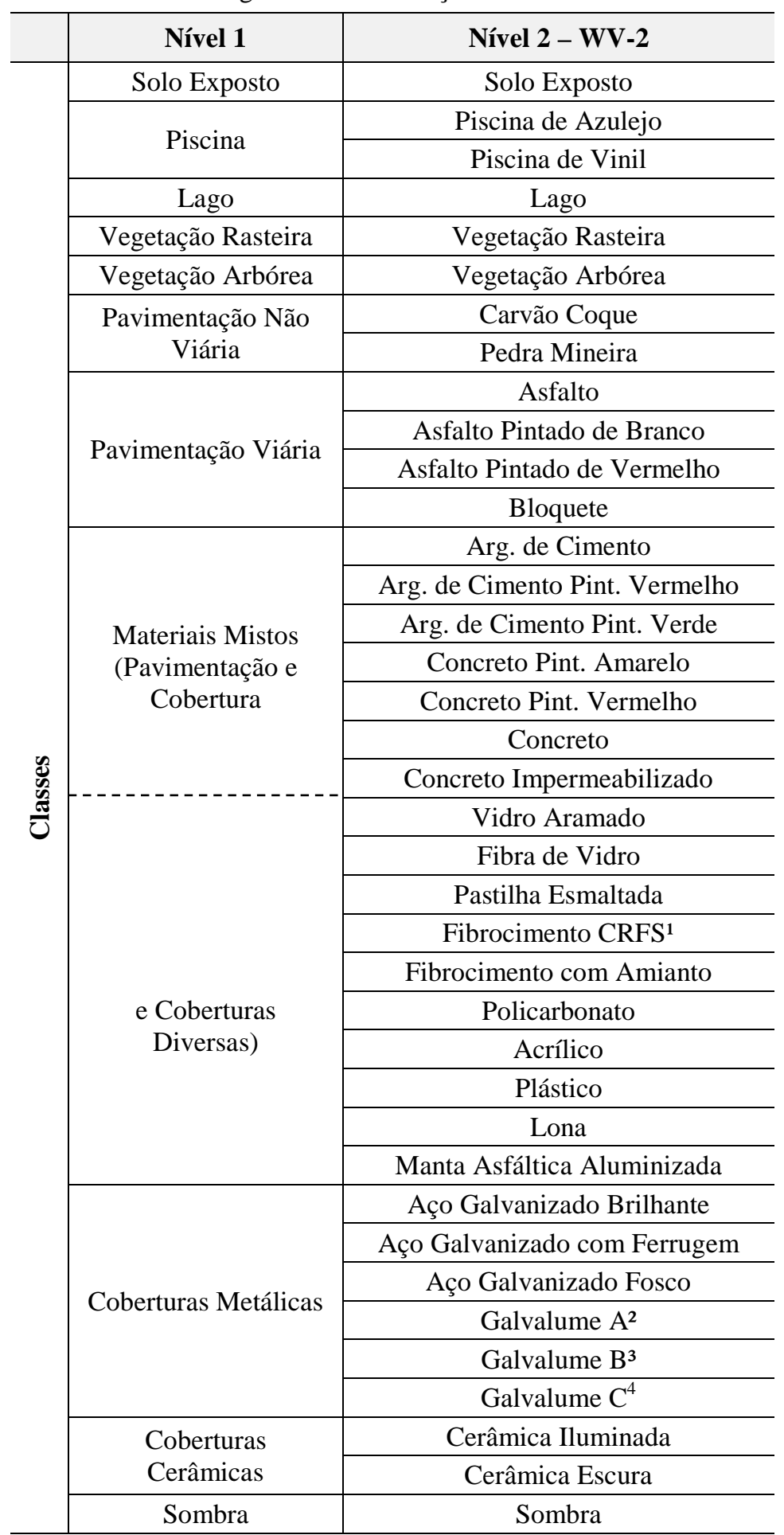

Cabe destacar o significado de alguns tipos de coberturas presentes na Tabela 2. A sigla CRFS, que significa Cimento Reforçado com Fios Sintéticos, corresponde a um tipo de telha de fibrocimento visivelmente semelhante às telhas de fibrocimento com amianto, sem possuí-lo em sua composição. Os diferentes tipos de galvalume representam coberturas metálicas compostas ou recobertas por camadas de alumínio, zinco e silício, que foram divididos em três tipos: A - ondulado com pintura eletrostática branca, B - trapezoidal sem pintura, $\mathrm{C}$ - ondulado sem pintura (Anjos, 2016).

O passo seguinte é a extração de amostras para a posterior mineração dos dados e a construção das árvores de decisão. As amostras foram importadas no formato CSV para o software WEKA (Waikato Environment for Knowledge Analysis), versão 
3.7.12 (University of Waikato, 2010). As amostras das classes com os atributos foram utilizadas como conjunto de treinamento para a indução das árvores de decisão e a classificação dos dados. O processo de mineração de dados desse software consiste na aplicação de algoritmos de Aprendizado de Máquina (Machine Learning) para a regressão, classificação, agrupamento ou seleção de atribu-tos dos dados, dentre outros (Hall et al., 2009).

\section{Algoritmos de Árvore de Decisão}

Os algoritmos de indução de árvores de decisão utilizados foram o Best First Tree BFTree (Shi, 2007) ou "Primeira Melhor Árvore" e Logistic Model Tree - LMT ou "Árvore de Modelo Logístico" (Landwehr et al., 2005). Estes algoritmos estão implementados no software WEKA.

O algoritmo de indução de árvores de decisão BFTree, proposto por Shi (2007), baseia-se na heurística best first, a qual utiliza o conhecimento sobre o domínio do problema para encontrar soluções mais eficientes do que no caso de busca cega (Witten \& Frank, 2000). Em cada nó interno da árvore, é utilizado apenas um atributo como teste. Tanto para atributos numéricos como categóricos, a divisão é binária. Os atributos numéricos são divididos por meio de limiares, enquanto 0 tratamento dos atributos categóricos é dependente do número de classes, isto é, em problemas de duas classes, em que os nós são representados por agrupamento em dois conjuntos, e em problemas de múltiplas classes, nos quais é utilizado um método heurístico de busca, que atinge um compromisso entre a redução da impureza e a velocidade de busca (Shi, 2007; Basgalupp, 2010).

O algoritmo LMT combina regressão logística e aprendizagem de árvore de decisão. A regressão logística é entendida como uma técnica estatística que objetiva produzir um modelo de predição de valores. A partir de um conjunto de observações, o modelo utiliza uma variável categórica, frequentemente binária, para representar uma série de variáveis contínuas e/ou binárias. Os modelos de regressão logística permitem selecionar os atributos relevantes de forma natural. Em comparação com as técnicas mais conhecidas de regressão, sobretudo a linear, a regressão logística distingue-se pelo fato de a variável resposta ser categórica (Landwehr et al., 2005; Witten \& Frank, 2000). Nesse contexto, a característica marcante da árvore de decisão gerada pelo algoritmo LMT é a sua estrutura com funções de regressão logística nas folhas. Possui em seus nós terminais, ao invés de classes únicas, vetores de variáveis $\left(x_{i}\right)$ e de coeficientes $\left(\beta_{j}\right)$ de todas as classes. Através desses valores, é possível calcular a função LMT, definida nas Equações 1 e 2.

$$
F(x)=x_{i}{ }^{\prime} \beta_{j}, \quad j=1, \ldots, J,
$$

em que:

$$
x_{i}{ }^{\prime} \beta_{j}=\sum_{k=0}^{K} \beta_{j k} x_{i k}
$$

sendo $x i 0=1$ e $x i$ e $\beta j$ correspondentes a vetores (coluna) de dimensão $(K+1)$, similar ao modelo logístico em estágio único.

\section{Avaliação dos Resultados}

A validação estatística dos resultados foi realizada por meio da utilização de mapas de verdade de campo, elaborado por Anjos (2016). A Figura 3 apresenta os mapas de verdade de campo nos dois níveis de detalhamento.

A fim de promover a validação dos resultados das classificações, foram gerados conjuntos de coordenadas aleatórias dentro dos limites da imagem utilizando o software Minitab 17 Statistical Software, com aproximadamente 2.000 pontos aleatórios para cada conjunto de validação do Nível 1 e 3.100 pontos aleatórios para cada conjunto de validação do Nível 2, sendo cada um deles produzido individualmente para cada classificação. Cada conjunto de coordenadas foi importado para o software QGis 2.14.2 (QGIS, 2009), e para cada ponto aleatório foi realizado o cruzamento espacial entre o mapa de verdade de campo e a classificação. O resultado é uma tabela de atributos associando cada ponto aleatório à classe de referência e àquela atribuída pela classificação. Assim, foi possível a geração 
das matrizes de confusão para o cálculo da exatidão global, índice Kappa, índice Kappa condicional, acurácias do produtor e usuário para cada classe (Congalton \& Green, 1999).

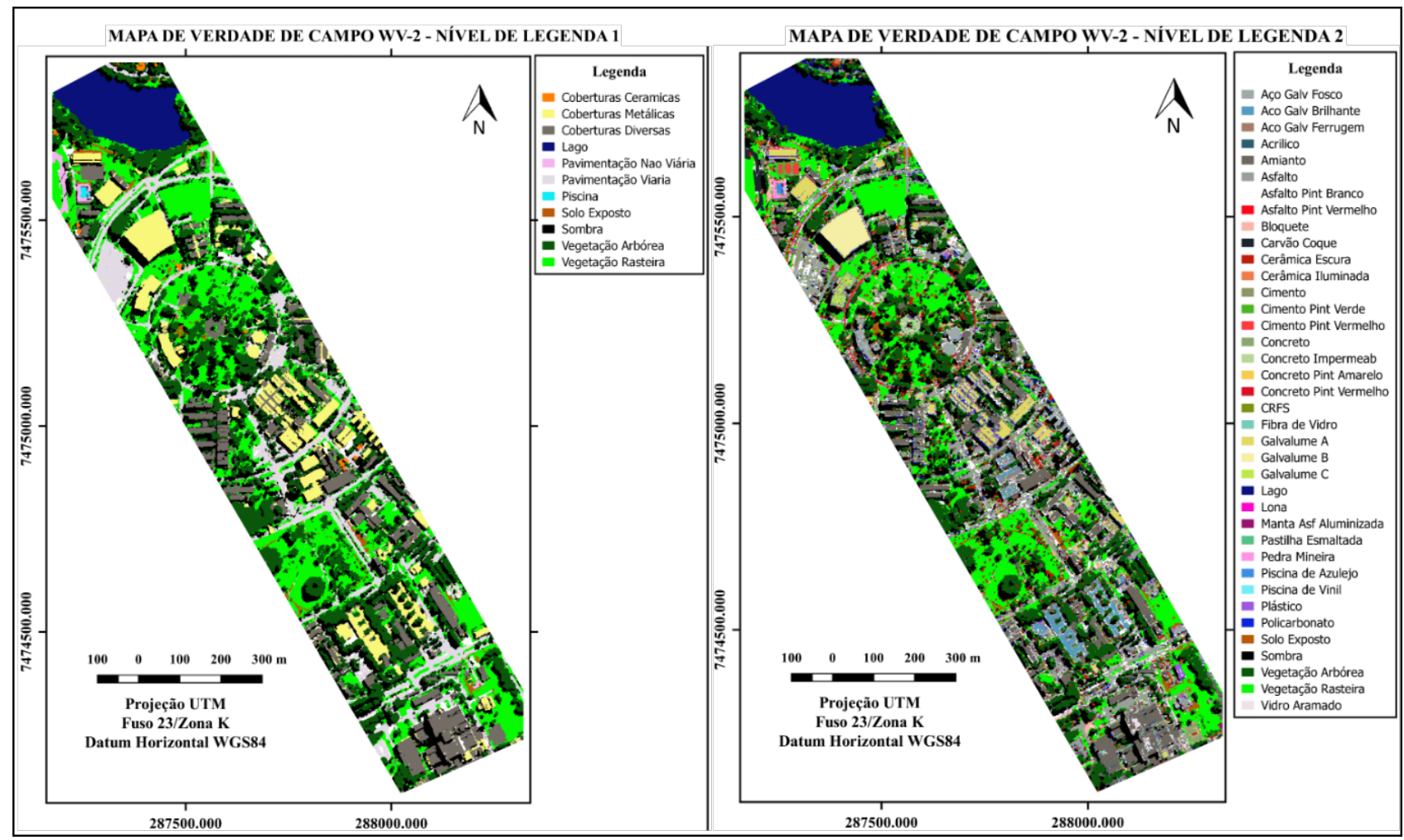

Figura 3 - Mapas de verdade de campo da área de estudo para ambos os níveis de legenda (Fonte: Anjos, 2016).

A fim de categorizar o índice Kappa, Landis \& Koch (1977) apresentam uma tabela com essa finalidade (Tabela 3). No entanto, os autores advertem que as divisões são arbitrárias, mas são úteis como padrões de referência para a discussão dos resultados.

Para finalizar a avaliação da acurácia das classificações, os resultados foram comparados entre si por meio do teste de significância estatística. Tal procedimento é realizado a fim de verificar se há diferença significante entre os índices Kappa das classificações dentro do intervalo de confiança considerado (Congalton \& Green, 2009; Furtado et al., 2013).

Tabela 3. Categorização do Índice Kappa.

\begin{tabular}{c|c}
\hline Valores & Categorização \\
\hline$<\mathbf{0}$ & Péssima \\
\hline $\mathbf{0}-\mathbf{0 , 2}$ & Ruim \\
\hline $\mathbf{0 , 2 1}-\mathbf{0 , 4}$ & Razoável \\
\hline $\mathbf{0 , 4 1}-\mathbf{0 , 6}$ & Bom \\
\hline $\mathbf{0 , 6 1}-\mathbf{0 , 8}$ & Muito Bom \\
\hline $\mathbf{0 , 8 1}-\mathbf{1 , 0}$ & Excelente
\end{tabular}

Fonte: Landis \& Koch (1977).

\section{RESULTADOS}

\section{Resultados para o Nível de Legenda 1}

A classificação final utilizando o algoritmo BFTree para o Nível de Legenda 1 é apresentada na Figura 4. Os atributos mais utilizados na construção de sua árvore de decisão foram SAVI e o NDVI 1. Esta classificação apresentou índice Kappa de 0,7068 e exatidão global de 75,03\%.

A classificação final utilizando o algoritmo LMT para o Nível de Legenda 1 é apresentada na Figura 5. Os atributos mais utilizados foram NDVI 1 e AC 3. Esta classificação apresentou índice Kappa de 0,7549 e exatidão global de 79,28\%.

\section{Resultados para o Nível de Legenda 2}

A classificação final utilizando o algoritmo BFTree para o Nível de Legenda 2, com 38 classes, é apresentada na Figura 6. $\mathrm{O}$ algoritmo selecionou 32 atributos na montagem da árvore de decisão, tendo sido reconhecidos como mais frequentes: o SAVI e o NDVI 1. Esta classificação apresentou índice Kappa de 0,6977 e exatidão global de 72,44\%. 


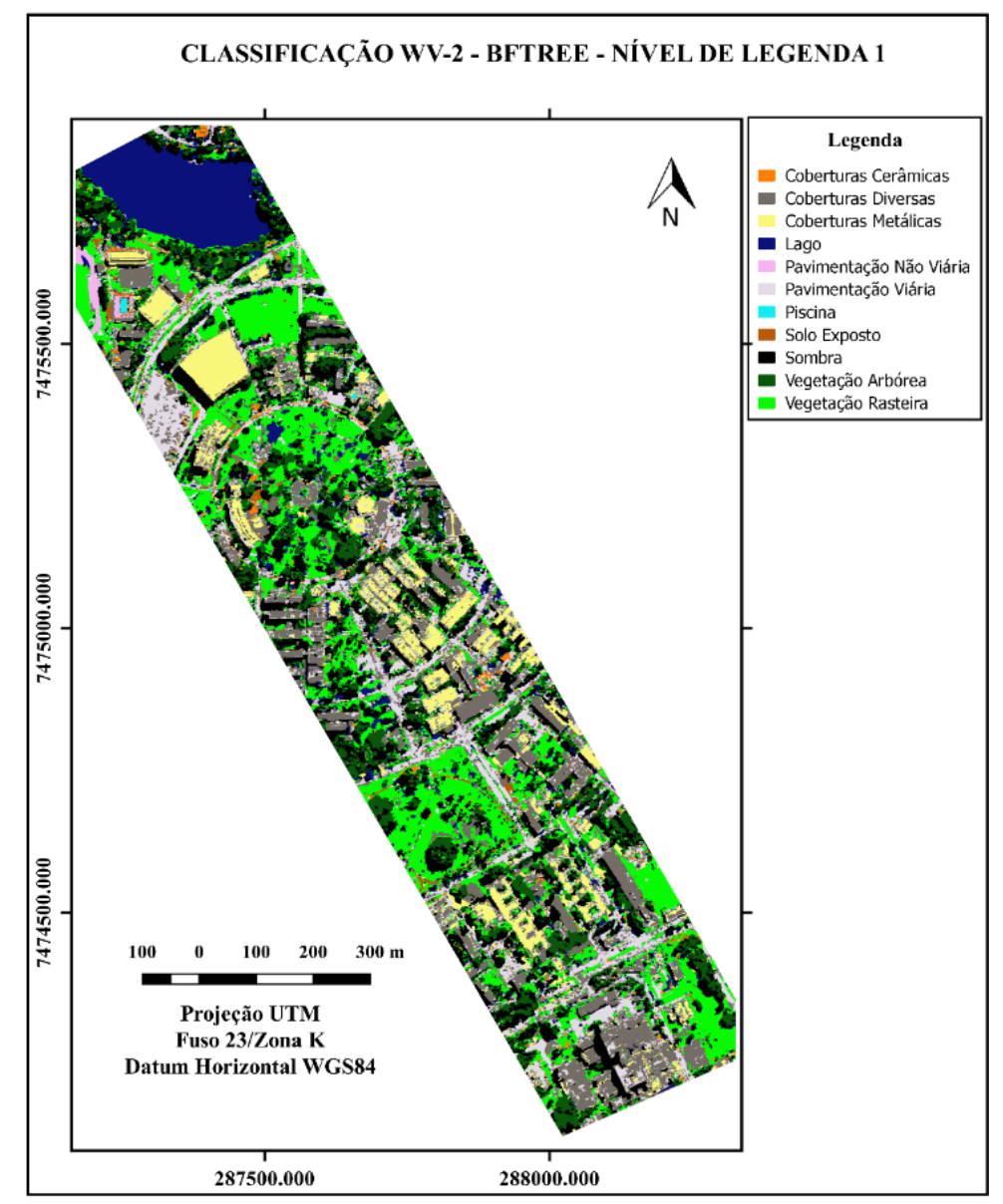

Figura 4 - Classificação da imagem WV-2 com o algoritmo BFTree para o Nível de Legenda 1.

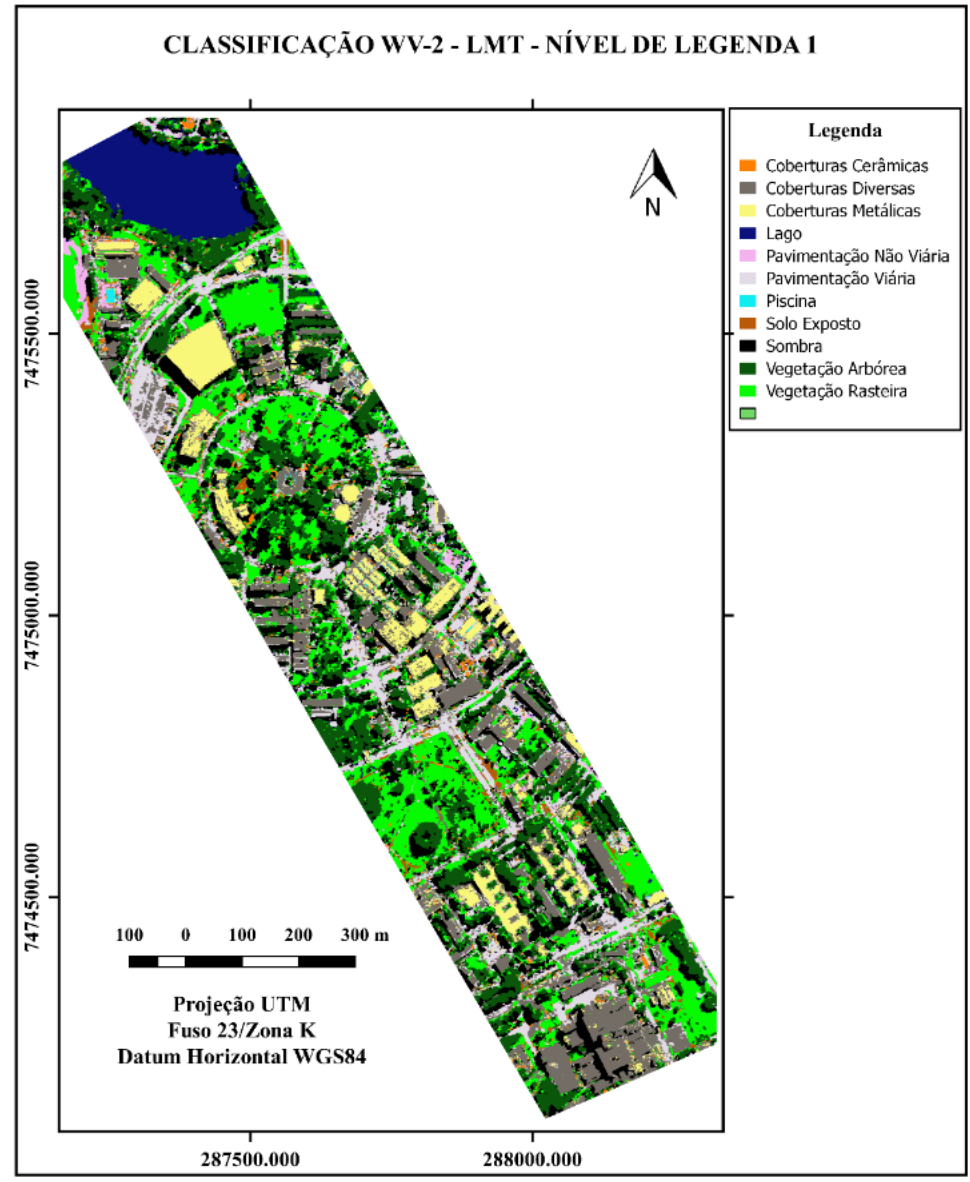

Figura 5 - Classificação da imagem WV-2 com o algoritmo LMT para o Nível de Legenda 1. 


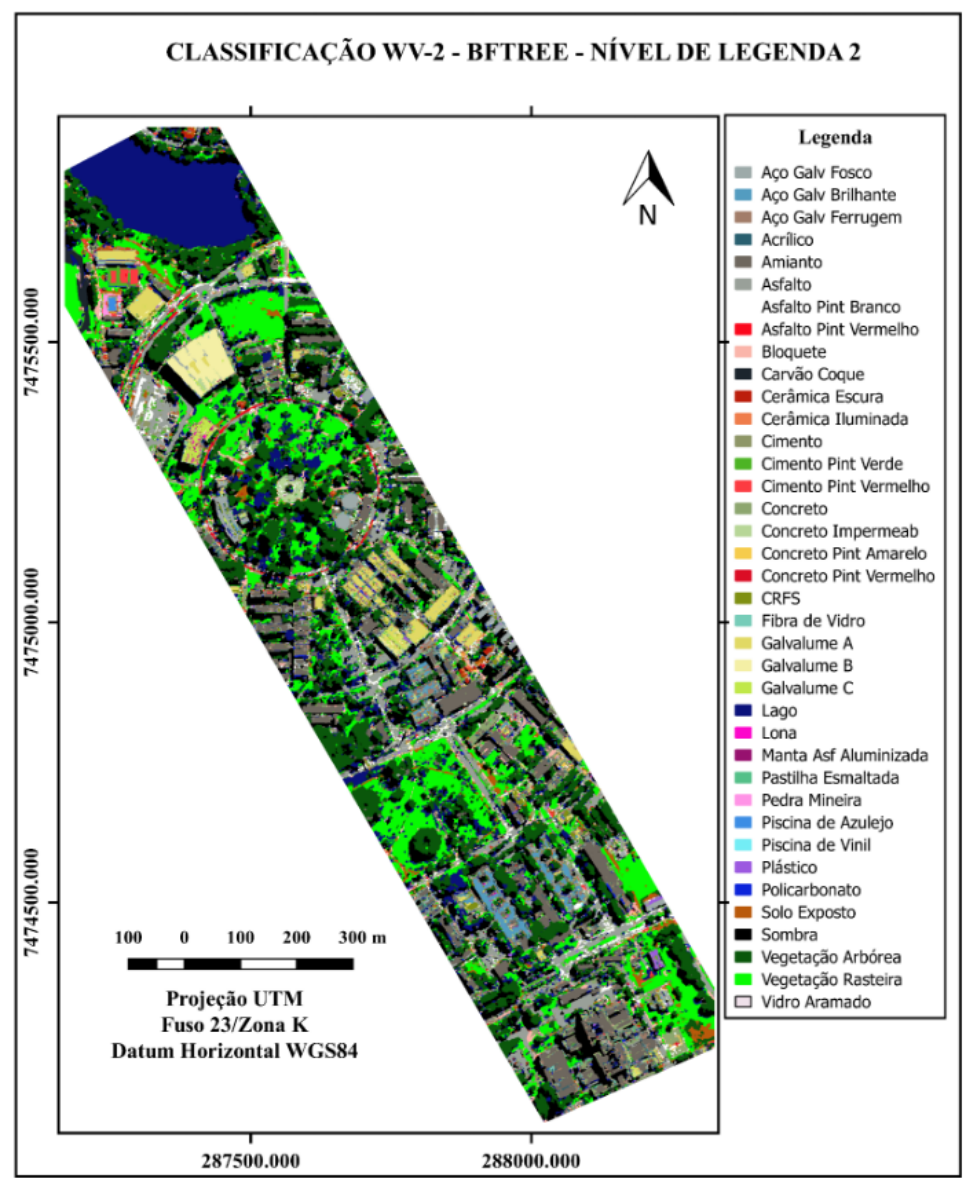

Figura 6 - Classificação da imagem WV-2 com o algoritmo BFTree para o Nível de Legenda 2.

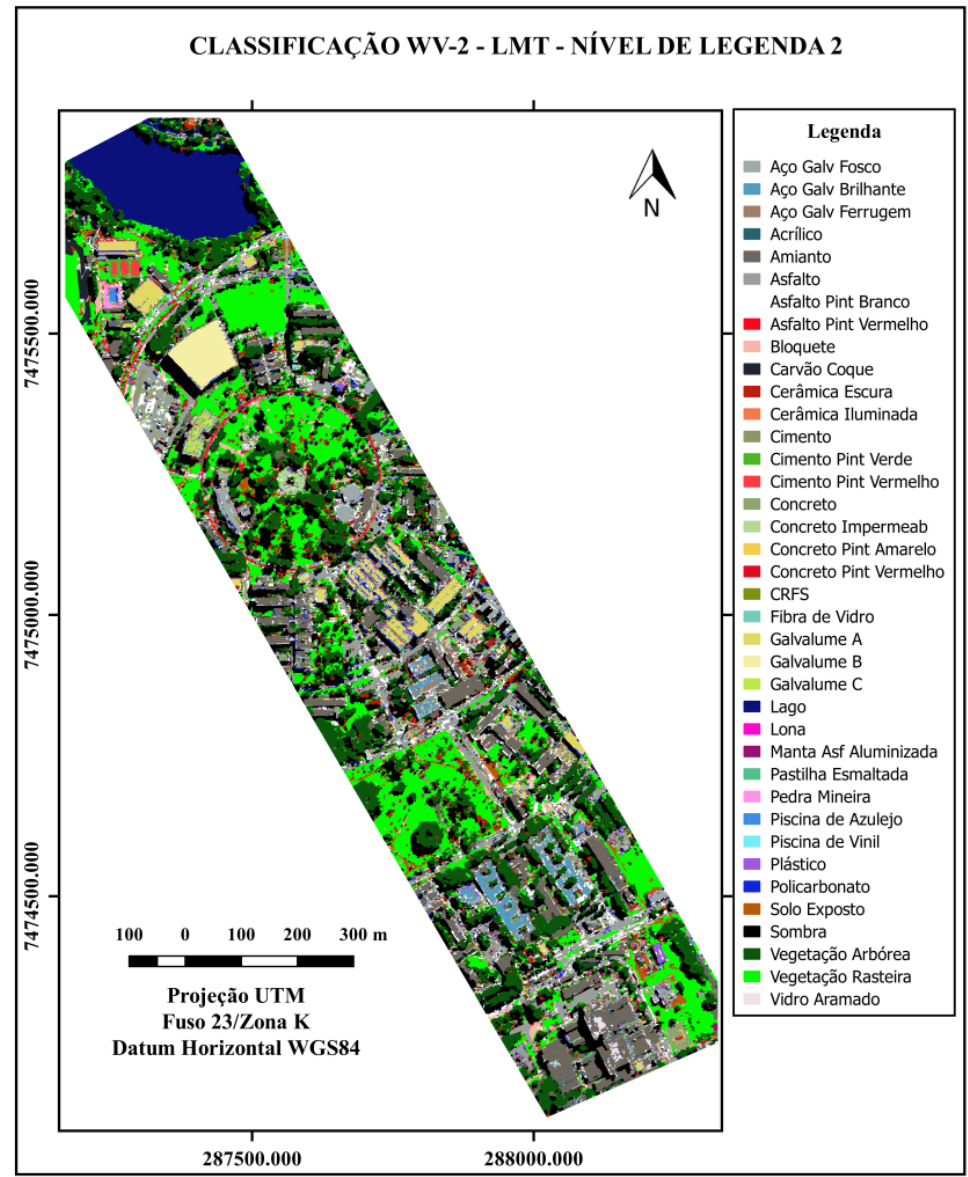

Figura 7. Classificação da imagem WV-2 com o algoritmo LMT para o Nível de Legenda 2. 
Tabela 3 - Índice Kappa condicional do usuário e produtor referente às classificações dos dados WV-2 para o Nível de Legenda 1.

\begin{tabular}{c|c|c|c|c}
\hline \multirow{2}{*}{ Classes } & \multicolumn{2}{|c|}{ Algoritmo BFTree } & \multicolumn{2}{c}{ Algoritmo LMT } \\
\cline { 2 - 5 } & $\mathbf{K}_{\mathbf{u}}$ & $\mathbf{K}_{\mathbf{p}}$ & $\mathbf{K}_{\mathbf{u}}$ & $\mathbf{K}_{\mathbf{p}}$ \\
\hline Coberturas Cerâmicas & 0,2050 & 0,4916 & 0,0615 & 0,1551 \\
\hline Coberturas Metálicas & 0,8109 & 0,7775 & 0,9118 & 0,7539 \\
\hline Coberturas Diversas & 0,5525 & 0,8517 & 0,5699 & 0,8395 \\
\hline Lago & 0,6964 & 1,0000 & 0,9671 & 1,0000 \\
\hline Pavimentação Não Viária & 0,5684 & 1,0000 & 0,2902 & 0,8307 \\
\hline Pavimentação Viária & 0,7876 & 0,7092 & 0,7237 & 0,6812 \\
\hline Piscina & 0,4987 & 0,3322 & 1,0000 & 1,0000 \\
\hline Solo Exposto & 0,7959 & 0,5154 & 0,4543 & 0,6408 \\
\hline Sombra & 0,6098 & 0,6422 & 0,8003 & 0,6805 \\
\hline Vegetação Arbórea & 0,8271 & 0,4404 & 0,7723 & 0,7158 \\
\hline Vegetação Rasteira & 0,7199 & 0,7749 & 0,7691 & 0,7175 \\
\hline
\end{tabular}

A classificação final utilizando o algoritmo LMT para o Nível de Legenda 2, com 38 classes, é apresentada na Figura 8. O algoritmo utilizou com maior frequência os atributos SAVI, NDVI 1, NDVI 2 e AC17. Esta classificação apresentou índice Kappa de 0,7210 e exatidão global de 74,37\%.

Resultados dos Índices Kappa Condicionais e Testes de Hipótese

As Tabelas 3 e 4 apresentam os valores do índice Kappa condicional por classe para as classificações realizadas.

Testes de hipóteses $Z$ com nível de significância de $5 \%$ foram realizados para comparar as classificações. As comparações dos resultados em ambos os níveis comprovaram que o índice Kappa obtido pelo algoritmo LMT é significantemente maior que o índice Kappa obtido pelo algoritmo BFTree.

\section{DISCUSSÕES}

As classes que apresentaram menores índices Kappa condicionais e proporcionam maiores confusões nas classificações foram aquelas que representam materiais visualmente semelhantes. Esta característica é corroborada pela presença de classes muito similares, sobretudo no Nível de Legenda 2, destacandose: diferentes tipos de aço galvanizado, de galvalumes, de amianto, concreto, asfalto. Também, destacam-se as confusões geradas entre classes espectralmente semelhantes, como cerâmica com solo exposto e policarbonato intemperizado com asfalto.

Os algoritmos utilizados neste trabalho apresentam características diferentes. Enquanto o BFTree corresponde a uma árvore de decisão clássica, o LMT consiste em uma árvore de decisão logística, onde seus nós terminais correspondem a funções logísticas para a identificação das classes. Neste sentido, os atributos utilizados foram diferentes, bem como a quantidade dos mesmos selecionada por cada algoritmo.

O algoritmo BFTree utilizou mais atributos para gerar as árvores de decisão no Nível de Legenda 2, pois a quantidade de classes a serem identificadas impacta decisivamente essa característica.

Em geral, o algoritmo LMT criou uma árvore de decisão com somente um modelo logístico para identificar as classes. O número de atributos utilizados nas funções logísticas de cada classe é variável, mas em média são utilizados de sete a oito atributos para a identificação de uma classe.

Os testes de hipótese comparando o desempenho dos algoritmos indicaram que $o$ algoritmo LMT apresentou resultado de índice Kappa superior ao BFTree em todos os níveis de dados. Essa superioridade apresentada pelo algoritmo LMT reside na utilização de um modelo de regressão logística ao invés de regressão linear (caso do BFTree) para selecionar os atributos mais relevantes no conjunto de dados. Com isso, é possível obter a estimativa das probabilidades das classes ao invés de simplesmente obter uma única classe no nó terminal da árvore de decisão (Landwehr et al., 2005). 
Tabela 4. Índice Kappa condicional do usuário e produtor referente às classificações dos dados WV-2 para o Nível de Legenda 2.

\begin{tabular}{|c|c|c|c|c|}
\hline \multirow{2}{*}{ Classes } & \multicolumn{2}{|c|}{ Algoritmo BFTree } & \multicolumn{2}{|c|}{ Algoritmo LMT } \\
\hline & $\mathbf{K}_{\mathbf{u}}$ & $\mathbf{K}_{\mathbf{p}}$ & $\mathbf{K}_{\mathbf{u}}$ & $\mathbf{K}_{\mathbf{p}}$ \\
\hline Aço Galvan. Brilhante & 0,4191 & 0,7957 & 0,3618 & 0,6061 \\
\hline Aço Galvan. Ferrugem & 0,7456 & 0,0753 & 0,4981 & 0,3316 \\
\hline Aço Galvan. Fosco & 0,6350 & 0,5819 & 0,5817 & 0,7618 \\
\hline Acrílico & 0,1562 & 0,0331 & 0,4975 & 0,3311 \\
\hline Amianto & 0,7818 & 0,8565 & 0,8215 & 0,7529 \\
\hline Asfalto & 0,6985 & 0,7989 & 0,7946 & 0,6317 \\
\hline Asfalto Pint. Branco & 0,3849 & 0,3849 & 0,2504 & 0,1662 \\
\hline Asfalto Pint. Vermelho & 0,6109 & 0,6109 & 0,8302 & 0,9072 \\
\hline Bloquete & 0,4891 & 0,1002 & 0,3510 & 0,2504 \\
\hline Carvão Coque & 0,9696 & 0,8763 & 0,8913 & 0,6660 \\
\hline Cerâmica Escura & 0,2426 & 0,3995 & 0,6822 & 0,1979 \\
\hline Cerâmica Iluminada & 0,4601 & 1,0000 & 0,4154 & 1,0000 \\
\hline $\begin{array}{l}\text { Arg. de Cimento } \\
\end{array}$ & 0,7755 & 0,3016 & 1,0000 & 0,1990 \\
\hline Arg. de Cimento Pint. Verde & 1,0000 & 0,2793 & 0,8079 & 0,8487 \\
\hline Arg. de Cimento P. Vermelho & 0,7979 & 0,3449 & 1,0000 & 0,9159 \\
\hline \begin{tabular}{|c|} 
Concreto \\
\end{tabular} & 0,6443 & 0,4410 & 0,7368 & 0,5764 \\
\hline Concreto Impermeab. & 0,6774 & 0,8220 & 0,7890 & 0,9458 \\
\hline Concreto Pint. Amarelo & 0,8993 & 0,5605 & 0,8448 & 0,5475 \\
\hline Concreto P. Vermelho & 0,3304 & 0,0988 & 1,0000 & 0,3481 \\
\hline CRFS & 0,4410 & 0,2828 & 0,4983 & 0,7487 \\
\hline Fibra de Vidro & 0,7201 & 0,7628 & 0,7970 & 0,9128 \\
\hline Galvalume A & 0,7437 & 0,9250 & 0,8413 & 0,9008 \\
\hline Galvalume B & 0,9519 & 0,9519 & 0,9469 & 0,8992 \\
\hline Galvalume C & 0,6659 & 0,7995 & 0,3742 & 1,0000 \\
\hline Lago & 1,0000 & 0,3224 & 1,0000 & 0,8567 \\
\hline Lona & 0,2844 & 1,0000 & 0,1959 & 0,1631 \\
\hline Manta Asf. Alum. & 0,8880 & 0,8880 & 0,7261 & 0,7991 \\
\hline Pastilha Esmaltada & 0,4982 & 0,1242 & 0,5986 & 0,3737 \\
\hline Pedra Mineira & 0,5157 & 1,0000 & 0,6471 & 0,7501 \\
\hline Piscina de Azulejo & 0,9150 & 1,0000 & 0,7731 & 1,0000 \\
\hline Piscina de Vinil & 0,5546 & 1,0000 & 0,7995 & 0,6660 \\
\hline Plástico & 0,7302 & 0,4192 & 0,9493 & 0,5551 \\
\hline Policarbonato & $-0,0081$ & $-0,0063$ & 0,0256 & 0,0157 \\
\hline Solo Exposto & 0,4253 & 0,1498 & 0,6351 & 0,4947 \\
\hline Sombra & 0,6936 & 0,8832 & 0,6225 & 0,8503 \\
\hline Vegetação Arbórea & 0,7596 & 0,9096 & 0,7012 & 0,8601 \\
\hline Vegetação Rasteira & 0,6215 & 0,9022 & 0,6444 & 0,8386 \\
\hline Vidro Aramado & 0,4123 & 0,7451 & 0,9873 & 0,8862 \\
\hline
\end{tabular}

Quanto ao processo de poda, ambos os algoritmos utilizam a validação cruzada. A validação cruzada é utilizada para combinar o crescimento da árvore com a seleção do número de expansões realizadas. Mesmo utilizando processos de poda baseados em um mesmo conceito, as árvores de decisão geradas pelo algoritmo LMT são bem menores quando comparadas com o algoritmo BFTree. Landwehr et al. (2005) credita as maiores acurácias geradas pelas classificações utilizando o algoritmo LMT ao processo de poda, por este utilizar validação cruzada, bem como a adoção do uso de modelos de regressão logística.

Outro aspecto em relação ao desempenho 
dos algoritmos é a diferença dos índices Kappa interníveis. Ambos os algoritmos obtiveram melhores índices Kappa no Nível de Legenda 1, pois este nível contempla apenas onze classes de cobertura do solo. Ao passo que o número de classes aumenta, caso do Nível de Legenda 2, o desempenho dos algoritmos sofre uma piora significante.

Qualquer algoritmo apresenta dificuldades para identificar um número elevado de classes. O Nível de Legenda 2 contempla 38 classes. Aí está a justificativa para o menor desempenho de ambos algoritmos no nível de detalhamento mais refinado.

\section{CONCLUSÕES}

O presente trabalho comparou classificações de cobertura do solo urbano a partir de uma cena WorldView-2 utilizando os algoritmos de árvore de decisão BFTree e LMT em dois níveis de legenda de classificação, respectivamente com 11 e 38 classes de cobertura do solo. As classificações para ambos os níveis apresentaram exatidão global acima de 70\% e índices Kappa oscilando entre 0,7068 e 0,7863 no Nível de Legenda 1, e entre 0,6977 e 0,7210 no Nível de Legenda 2. Os testes de hipótese demonstraram que os resultados alcançados pelo algoritmo LMT foram significantemente superiores aos resultados obtidos pelo algoritmo BFTree.

Os resultados alcançados comprovam que as imagens orbitais que conciliam altas resoluções espaciais e espectrais são adequadas para a identificação das classes de cobertura do solo urbano em nível de legenda extremamente detalhado. Também, a utilização de algoritmos de mineração de dados, como as árvores de decisão, colabora efetivamente na melhora da acurácia destas classificações.

\section{AGRADECIMENTOS}

Os autores gostariam de expressar a sua gratidão às empresas DigitalGlobe, na pessoa do Sr. Roberto Varela, e Satmap, na pessoa do Sr. Weber Pires, pela gentil cessão da imagem WorldView-2. Da mesma forma, expressam seus agradecimentos a CAPES pela concessão da bolsa de mestrado ao primeiro autor, cuja pesquisa de dissertação se encontra sumariada neste artigo.

\section{REFERÊNCIAS}

ANJOS, C.S. Classificação de áreas urbanas com imagens multiespectrais e hiperespectrais utilizando métodos não-paramétricos. São José dos Campos, 2016. Tese (Doutorado em Sensoriamento Remoto), Instituto Nacional de Pesquisas Espaciais.

BASGALUPP, M.P. LEGAL-Tree: um algoritmo genético multi-objetivo lexicográfico para indução de árvores de decisão. São Carlos, 2010. Tese (Doutorado em Ciências da Computação e Matemática Computacional), Instituto de Ciências Matemáticas e Computação (ICMC-USP).

BAUSCH, W.C. \& DUKE, H.R. Remote sensing of plant nitrogen status in corn. Transactions of the ASAE, v. 39, n. 5, p. 1869-1875, 1996.

BRASIL. Estatuto da Cidade e Legislação Correlata. Lei ${ }^{\circ}$ 10.257, de 10 de junho de 2001 - 2. ed.-Brasília: Senado Federal, Subsecretaria de Edições Técnicas, 80p., 2002. ISBN 85-7018-223-6

CONGALTON, R.G. \& GREEN, K. Assessing the Accuracy of Remotely Sensed Data: Principles and Practices. Boca Raton: Lewis Publishers, 1999.

CONGALTON, R.G. \& GREEN, K. Assessing the accuracy of remotely sensed data: principles and practices. 2 ed. Boca Raton: CRC Press, 183p., 2009.

DEFINIENS. Definiens Developer 7: Reference Book. Munique: Definiens AG, 2007. 195 p. Disp. em: http://www.ecognition.cc/download/ReferenceBook.pdf.

DIGITALGLOBE. WorldView-2 Data Sheet. 2009. 2 p. Disp. em: <https://www.digitalglobe.com/sites/default/files/DG _WorldView2_DS_PROD.pdf $>$.

SULLSOFT. Guia do ENVI em Português. Sulsoft, 2000. Disp.em www.sulsoft.com.br. Acesso em 15 out. 2006.

FURTADO, L.F.A.; FRANCISCO, C.N.; ALMEIDA, C.M.
Análise de Imagem Baseada em Objeto para Classificação das Fisionomias da Vegetação em Imagens de Alta Resolução Espacial. Geociências, São Paulo, v. 32, n. 3, p. 441-451, 2013.

GEOMATICA. PCI Geomatics Announces Release of Geomatica 2014. GATINEAU, Quebec, Canada: [s.n.], 2014. 5p. Disp. em: <http://www.pcigeomatics.com/pressnews/2014.

GITELSON, A.A.; KAUFMAN, Y.J.; MERZLYAK, M.N. Use of a green channel in remote sensing of global vegetation from EOS-MODIS. Remote Sensing of Environment, v. 58, n. 3, p. 289-298, 1996.

HALL, M.; FRANK, E.; HOLMES, G.; PFAHRINGER, B.; REUTEMANN, P.; WITTEN, I. H. The WEKA data mining software. [S.l.: s.n.], 10p., 2009.

HEROLD, M.; ROBERTS, D.A.; GARDNER, E.M.; DENNISON, P.E. Spectrometry for urban area remote sensing - Development and analysis of a spectral library from 350 to $2400 \mathrm{~nm}$. Remote Sensing of Environment, v. 91, p. 304-319, 2004

HUETE, A.R. A soil-adjusted vegetation index (SAVI). Remote Sensing of Environment, v. 25, p. 53-70, 1988.

IBGE. Atlas do Censo Demográfico 2010. Rio de Janeiro: IBGE, 2013. 156 p.

IBGE. Cidades@ - Consulta por Município. 2014. Disp. em: $<$ http://cod.ibge.gov.br/232TL>.

JAIN, S.K.; SARAF, A.K.; GOSWAMI, A.; AHMAD, T. Flood inundation mapping using NOAA AVHRR data. Water Resources Management, v. 20, n. 6, p. 949-959, 2006.

JENSEN, J.R. Sensoriamento remoto do ambiente: uma perspectiva em recursos terrestres. São José dos Campos: Parêntese, 2011. Tradução José Carlos Neves Epiphanio (coord.). 
LANDIS, J.R. \& KOCH, G.G. The measurement of observer agreement for categorical Data. Biometrics, v. 33, n. 1, p. 159-174, 1977.

LANDWEHR, N.; HALL, M.; FRANK, E. Logistic model trees. Mach. Learn, v. 59, n. (1-2), p. 161-205, 2005.

LEONARDI, F. Abordagens cognitivas e mineração de dados aplicadas a dados ópticos orbitais e de laser para classificação de cobertura do solo urbano. São José dos Campos - SP, 2010. Dissertação (Mestrado), Instituto Nacional de Pesquisas Espaciais.

QGIS Development Team, QGIS Geographic Information System. Open Source Geospatial Foundation, 2009. URLhttp://qgis.osgeo.org.

RICHARDSON, A.J. \& WIEGAND, C. Distinguishing vegetation from soil background information. Photogrammetric Engineering and Remote Sensing, v. 43, p. 1541-1552, 1977.

ROUSE, J.W.; HAAS, R.H.; SCHELL, J.A.; DEERING, D.W.; HARLAN, J.C. Monitoring the vernal advancement of retrogradation of natural vegetation. Greenbelt: NASA/GSFC, 371p., 1974.

SARKAR, A.; GARG, R.D.; SHARMA, N. RS-GIS Based Assessment of River Dynamics of Brahmaputra River in
India. Journal of Water Resource and Protection, v. 4, n. 02, p. 63-72, 2012.

SHI, H. Best-first decision tree learning. Hamilton, NZ, 2007. Master's thesis. University of Waikato.

TRIMBLE. eCognition Developer 8.7 User Guide. Munich, Germany: [s.n.], 2011. 258p. Disp. em: $<$ http://www.ecognition.com/>.

UNICAMP. A Unicamp. 2016. Disp. em: < http://www. unicamp.br/unicamp/a-unicamp>.

UNIVERSITY OF WAIKATO. Weka 3.7.12 - Machine Learning Software in Java. Disp. no site da University of Waikato 2010. URL: http://www.cs.waikato.ac.nz/ml/weka.

UNPD. World Urbanization Prospects: The 2014 Revision. [sn], 2014. 493p. Disp. em: http://esa.un.org/unpd/wup/ Publications/Files/WUP2014-Report.pdf $>$.

WITTEN, I.H. \& FRANK, E. Data mining: Practical machine learning tools and techniques with JAVA implementations. San Francisco: Morgan Kaufmann, 371p., 2000.

Submetido em 27 de julho de 2017 Aceito em 5 de março de 2018 